\title{
Altersgerechte Arbeit und Führung im digitalen Zeitalter
}

\author{
M. Falkenstein und C. Kardys
}

\section{Einleitung}

Im Zuge der demografischen Entwicklung steigt der Anteil älterer Beschäftigter stetig an. Zudem fordert die rasante digitale Umstrukturierung der Arbeit - die durch eine hohe Dynamik und Komplexität geprägt ist - den Beschäftigten ein hohes Maß an Motivation, Lernfähigkeit und mentalen („kognitiven“) Fähigkeiten ab. Solche Fähigkeiten, die auch als fluide Intelligenz bezeichnet werden, ändern sich jedoch mit zunehmendem Alter. Bislang wird im betrieblichen Kontext der Förderung der kognitiven Leistungsfähigkeit eine zu geringe Aufmerksamkeit geschenkt. Hier sollten arbeitsweltbezogene Maßnahmen ergriffen werden, um die Leistungs- und Beschäftigungsfähigkeit sowie die Gesundheit und das Wohlbefinden einer alternden Belegschaft zu erhalten und zu fördern, damit sie den ansteigenden Anforderungen gewachsen ist. Hierfür stehen im Rahmen des Arbeits- und Gesundheitsschutzes sowie des betrieblichen Gesundheitsmanagements zwei Ansätze zur Verfügung: Einerseits die verhaltenspräventive Ebene, die auf den Erhalt und die Förderung von persönlichen Kompetenzen und gesundheitlichen Faktoren von Beschäftigten abzielt, und andererseits verhältnispräventive Maßnahmen, die eine alternsfreundliche Gestaltung der Arbeit beinhalten. Verhaltenspräventive Interventionen für ältere Beschäftigte wurden von den Autoren bereits in verschiedenen Buchbeiträgen erörtert (z.B. BAuA 2012; Falkenstein, Gajewski \& Kardys 2017; Falkenstein 2017; Falkenstein et al. 2020; Kardys et al. 2020). Daher konzentriert sich das vorliegende Kapitel auf strukturelle Maßnahmen (Verhältnisprävention), die vor allem eine alters- und alternsgerechte Arbeit und Führung im Kontext moderner digitalisierter Arbeitswelten fokussieren und zukünftig an Bedeutung gewinnen müssen.

\section{Alternsbegleitende Veränderung von Fähigkeiten}

Die menschliche Arbeit wird durch das Zusammenspiel einer Vielzahl von Fähigkeiten („Funktionen“) ermöglicht. Die meisten dieser Funktionen 
verändern sich über die Lebensspanne. Insbesondere zeigt sich eine alternsbegleitende Abnahme der Sinnesfunktionen Sehen, Hören, Schmecken, Riechen und Tasten. Ferner verändert sich die Motorik: Die Muskelkraft schwindet, das Gleichgewicht und die Feinmotorik (v.a. bei neuen Aufgaben) nehmen ab. Insbesondere zeigen sich auch schon im mittleren Erwachsenenalter Beeinträchtigungen bestimmter mentaler („kognitiver“) Funktionen (Wild-Wall et al. 2009), u.a. lassen die Informationsverarbeitungsgeschwindigkeit, die flexible Steuerung der Aufmerksamkeit, die Unterdrückung unwichtiger Reize und falscher Aktionen sowie das Arbeitsgedächtnis nach. Diese Veränderungen kognitiver Funktionen verlaufen für Menschen gleichen Alters außerordentlich unterschiedlich und hängen von vielfältigen Faktoren ab. Die Betrachtung des Alters allein besitzt somit keine hohe Aussagekraft über die persönlichen Fähigkeiten, d.h. ältere Beschäftigte müssen individuell beurteilt werden.

Ein differenziertes Modell des Alterns geht also davon aus, dass zum einen verschiedene Fähigkeiten unterschiedlich altern (z.B. das Gedächtnis stark, die Konzentration jedoch kaum), zum anderen Veränderungen individuell höchst unterschiedlich sein können. Dies liegt am Einfluss verschiedenartiger interner (nicht beeinflussbarer) Faktoren wie der genetischen Ausstattung, sowie externer (in der Regel beeinflussbarer) Faktoren wie Bildung, Lebensstil, Stress, der Art der Arbeit, und nicht zuletzt der Vorurteile gegenüber Älteren (Falkenstein 2017). Die Art der Arbeit ist einer der wichtigsten externen Einflussfaktoren aufgrund der besonders langen „Einwirkzeit“ bei Berücksichtigung der durchschnittlichen Erwerbsjahre von 45 Jahren. In einer französischen Studie wurde bei über 2000 Beschäftigten die mentale Leistungsfähigkeit über einen Zeitraum von zehn Jahren erfasst, zudem der Grad der mentalen Stimulation durch die Arbeit. Es zeigte sich eine Verbesserung der mentalen Leistungsfähigkeit bei hoher kognitiver Stimulation durch die Arbeit, trotz Zunahme des Alters um zehn Jahre (Marquié et al. 2010). Im Rahmen des Projekts PFIFF konnte gezeigt werden, dass ältere Beschäftigte mit jahrelangen flexiblen Tätigkeiten, eine schwierige neue Aufgabe ähnlich gut wie jüngere Beschäftigte meistern konnten (Freude et al. 2010). Auf der anderen Seite ist seit Langem klar, dass monotone, geistig wenig anregende Arbeit Beschäftigte vorzeitig altern lässt (Hacker 2004). Geistig anregende Arbeit fördert also die mentale Fitness; monotone, wenig herausfordernde Arbeit sowie Schichtarbeit und vor allem Nachtarbeit hingegen beeinträchtigen sie.

Neben den beruflichen Tätigkeiten können Belastungen und Stress mentale Fähigkeiten Gesundheit und Wohlbefinden der Beschäftigten verringern (Falkenstein \& Kardys 2018). Als wesentliche arbeitsbezogene 
Stressoren gelten Zeit- und Leistungsdruck, Multitasking, häufige Unterbrechungen, geringe Kontrolle über die Arbeit, unfaire Entlohnung, Monotonie und Arbeitsplatzunsicherheit sowie nicht zuletzt Mobbing und schlechte Führung (Lohmann-Haislah 2013). Dauerhafter (chronischer) Stress wirkt sich negativ auf verschiedene kognitive Funktionen aus und erhöht das Risiko für Herz-Kreislauferkrankungen, Burnout und Depressionen. Vor allem ältere Beschäftigte zeigen starke negative Körperreaktionen auf Stress, falls sie nicht über hinreichende Bewältigungsstrategien verfügen (Otte \& Seifritz 2014).

Die Leistungsfähigkeit Älterer wird nicht zuletzt durch altersbezogene negative Vorurteile (sog. Altersstereotype) beeinflusst (z.B. Initiative Neue Qualität der Arbeit 2017). Stereotype wirken nicht zwangsläufig, sondern sie werden meist unbewusst durch bestimmte Hinweisreize aktiviert, wie eine Altersangabe oder graues Haar. Durch Altersstereotype wird nicht nur das Fremdbild von älteren Menschen bestimmt, sondern auch das Selbstbild Älterer und dadurch ihre Leistung beeinflusst. Beispielsweise können die Bereitschaft zur Beteiligung an Weiterbildung und sogar die Lernfähigkeit mit dem Alter abnehmen, wenn ein Älterer das Altersstereotyp „Ältere sind nicht mehr lernfähig" forciert.

Funktionelles Altern ist also weitgehend das Ergebnis von langen einwirkenden Umwelteinflüssen, die größtenteils beeinflussbar sind. Diese Einflüsse gilt es daher altersgerecht zu gestalten.

\section{Alters- und alternsgerechte Arbeitsgestaltung}

Altersgerechte Arbeitsgestaltung bedeutet die Anpassung von Arbeitsplatz und Arbeitsumgebung an die veränderten Fähigkeiten älterer Beschäftigter. Alternsgerechte Arbeitsgestaltung sorgt dafür, dass Beschäftigten gesund altern können und ihre Kompetenzen und ihre Arbeitsfähigkeit möglichst lange bewahren. Hierzu gehört eine adäquate Weiterbildung alternder Beschäftigter. Alle diese Maßnahmen sind nur auf der Grundlage einer kompetenten alter(n)sgerechten Führung möglich. Eine ausführliche Darstellung alters- und v.a. alternsgerechter Arbeitsgestaltung findet sich bei Mühlenbrock (2016). 


\section{Altersgerechte Arbeitsgestaltung}

Die ergonomische Gestaltung von Arbeitsplätzen sollte mögliche Problembereiche Älterer im Blick haben. Hierbei reicht es nicht, die Beleuchtung für ältere Beschäftigte zu verstärken oder das Heben großer Lasten zu vermeiden. Vielmehr müssen auch subtilere sensorische, motorische und v.a. kognitive Veränderungen Älterer bei der Arbeitsplatzgestaltung Berücksichtigung finden. Insbesondere kognitive Veränderungen sind hoch bedeutsam, da die kognitive Leistungsfähigkeit im Zuge der Digitalisierung zunehmend wichtiger wird (Wild-Wall et al. 2009; Falkenstein \& Kardys 2020).

Im Alter kommt es zu Einschränkungen von Körperfunktionen und Wahrnehmungsfunktionen, die eine altersfreundliche Arbeitsgestaltung berücksichtigen muss. Ältere haben oft erhebliche Schwierigkeiten beim Halten des Gleichgewichts. Hierdurch steigt die Gefahr von Stürzen und Unfällen. Geeignete Gegenmaßnahmen stellen z.B. auffällige und klare Kennzeichnung von Wegen und das Wegräumen von Hindernissen und Stolperfallen dar. Für das Erste sollte der Arbeitgeber*innen, für das Zweite der Arbeitnehmer*innen Sorge tragen.

Ältere haben zunehmend Schwierigkeiten mit Präzisionsbewegungen und -handlungen, wenn diese nicht eingeübt sind (Vieluf et al. 2020). Daher sollten ältere Beschäftigte neue Bewegungsabläufe, z.B. bei der Einführung neuer Technologien in einer längeren Einübungsphase trainieren.

Besonders bedeutsam sind Einschränkungen der Sinneswahrnehmung. Bildschirme sollten möglichst wenig reflektieren, da Ältere durch Blendung stärker beeinträchtigt werden. Leuchtdichte, Zeichengröße und Zeilenabstände sollten hinreichend groß sein.

Bei den mentalen (kognitiven Funktionen) zeigen Ältere zunehmend Probleme mit der flexiblen Steuerung der Aufmerksamkeit und der kognitiven Ressourcen (für eine Zusammenfassung siehe Wild-Wall et al. 2009). Ältere sind ablenkbarer als Jüngere und haben oft Probleme beim Suchen von Informationen (z.B. Wild-Wall et al. 2009; Getzmann et al. 2013). Daher sollten Arbeitsplätze sowie Beschilderungen und Benutzeroberflächen möglichst sparsam und übersichtlich gestaltet werden. Texte sollten möglichst prägnant sein und wichtige Informationen hervorheben. Auch akustische Störreize, v.a. eine erhöhte Geräuschkulisse in Großraumbüros lenken Ältere häufig stark ab. Sie sollten durch technische und/oder organisatorische Maßnahmen reduziert werden.

Ältere haben insbesondere Schwierigkeiten, mehrere Tätigkeiten zeitgleich auszuführen. Als geeignete Maßnahmen gelten die Vermeidung von Doppel- oder gar Mehrfachtätigkeiten in betrieblichen Abläufen. Da die 
Fehlerwahrnehmung bei Zeitdruck, hochkomplexen Aufgaben und Multitasking (Weißbecker-Klaus 2014) sowie bei Älteren generell (Falkenstein et al. 2001), abgeschwächt ist, sollten Ältere v.a. bei schwierigen Aufgaben nicht unter Zeitdruck gesetzt werden und die Möglichkeit einer sequenziellen Abarbeitung haben.

Bei nicht altersgerechter Arbeitsgestaltung kann es zu negativen Folgen für Ältere kommen. Kognitive Ressourcen müssen verstärkt investiert werden, um trotz schlechter/schwieriger Bedingungen die Leistung möglichst hoch zuhalten. Diese Ressourcen fehlen dann für die Arbeit, v.a. für komplexe digitalisierte und virtualisierte Arbeitsplätze. Zudem ermüden ältere Beschäftigte dadurch schneller. Diese Situation kann in Überlastung und Stress, mit den beschriebenen negativen Auswirkungen für die körperliche und geistige Fitness sowie Gesundheit münden.

Die für ältere Beschäftigte notwendigen Maßnahmen der Arbeitsgestaltung nützen auch Jüngeren und können daher als gute Ergonomie für alle gelten („design for all“-Prinzip).

Eine ausführlichere Beschreibung von Maßnahmen der altersgerechten Arbeitsgestaltung findet sich bei Falkenstein (2013).

\section{Alternsgerechte Arbeitsgestaltung und Weiterbildung}

Ungünstige Arbeitsbedingungen beeinträchtigen die mentale Kompetenz und die Gesundheit alternder Beschäftigter. Negative Einflussfaktoren sind Arbeitsmenge und Zeitdruck, Monotonie und langjährig konstante Arbeit, Unkontrollierbarkeit der Arbeitsabläufe und fehlende Handlungsspielräume, eine schlechte Passung von Arbeit und eigener Kompetenz, ungünstige Schichtarbeit und schlechtes Führungsverhalten. Die wesentlichen Maßnahmen zur alternsgerechten Arbeitsorganisation sind daher geistig anregende, abwechslungsreiche und ganzheitliche Arbeit, möglichst weite Entscheidungsspielräume, die Reduktion von Arbeitsmenge und Zeitdruck, die Passung von Anforderungen und Kompetenzen, und eine gute Gestaltung von Schichtarbeit. Bei durchgängig repetitiver Arbeit, (die häufig besteht), lässt sich die Monotonie durch den Wechsel zwischen Tätigkeiten (Rotation) verringern. Zur Erhöhung der Kontrollspielräume sollten nur Ziele und Termine gesetzt werden, um so die Eigenverantwortung und Flexibilität zu erhöhen; auch bei Routinearbeit müssen Möglichkeiten der Partizipation der Beschäftigten bei Einteilung von Arbeitsabläufen geschaffen werden. Hohe Handlungsspielräume und flexible Arbeit verbessern neuronale Funktionen und dadurch die mentale Kompetenz älterer Beschäftigter (Freude et al. 2010). Darüber hinaus führen sie zu einer bes- 
seren mentalen Gesundheit im Rentenalter und schützen vor Demenz (Rodriguez \& Spilski 2020). Auch mehrfache Arbeitswechsel fördern die mentale Fitness bis ins hohe Alter: Ältere Beschäftigte mit mehreren horizontalen Arbeitsplatzwechseln sind kognitiv fitter als Beschäftigte mit keinem oder nur einem Wechsel, was sich auch in neuronalen Strukturen zeigt (Godde et al. 2020). Mit ansteigendem Alter erhöht sich die Relevanz einer adäquaten Passung zwischen Qualifikationen und den Anforderungen des Arbeitsplatzes. Fehlanpassungen können Stress verursachen mit den bereits erwähnten kurz- und langfristigen Folgen. Gestufte Gegenmaßnahmen stellen a) die Erstellung eines Kompetenzprofils des Beschäftigten, b) der Abgleich zwischen Kompetenzprofil und Arbeitsplatz und c) persönliche und berufsbezogene Weiterbildung sowie Kompetenzentwicklung dar. Weiterbildungs- und Qualifizierungsmaßnahmen sollten alternsgerecht gestaltet werden (Falkenstein 2012). Dabei gilt es, Zeitdruck und Ablenkung stets zu vermeiden und die Besonderheiten des Lernens beim Alterungsprozess zu berücksichtigen. Hierzu sind Kenntnisse der funktionalen Stärken und Schwächen sowie der Motivationsstruktur in den verschiedenen Phasen über die Lebensspanne notwendig (Stamov Roßnagel 2008; Falkenstein 2012). Dennoch sollten möglichst selten spezielle Weiterbildungskurse beispielsweise nur für Jüngere oder nur für Ältere durchgeführt werden, da sie möglicherweise als diskriminierend empfunden und daher gemieden werden. Die Gestaltung von Weiterbildungsmaßnahmen für die Bedürfnisse Älterer (wie z.B. das Vermeiden von Ablenkung) nützt in der Regel auch Jüngeren (nach dem bereits genannten „design for all“Prinzip). Ältere haben jedoch eine spezifische Vorliebe für selbstgesteuertes Lernen. Daher sollte älteren Beschäftigten möglichst große Freiräume beim Lernen eingeräumt werden. Umstrukturierungen und die Einführung neuer Technologien sollten langfristig vorbereitet und die Betroffenen ohne Zeitdruck geschult werden, da sich Zeitdruck besonders bei Älteren nachteilig auf die mentale Leistungsfähigkeit auswirkt.

\section{Alternsgerechte Führung in analogen und virtuellen Teams}

Die wichtigste Aufgabe von Führungskräften ist das Management der Arbeitsfähigkeit der Beschäftigten (Ilmarinen \& Tempel 2013). Dies beinhaltet die Gestaltung von gesunder Arbeit und Arbeitsumgebung, die Förderung von Motivation und Kompetenz der Beschäftigten.

Führungskräfte sind unerlässlich, um die entscheidenden Weichen bei der oben beschriebenen alter(n)sgerechten Arbeitsgestaltung $\mathrm{zu}$ stellen. Die Herausforderung liegt v.a. darin, das eigene Unternehmen (samt Un- 
ternehmens- und Führungskultur) zu befähigen, sich für die Arbeitsweltveränderungen nicht nur zu öffnen, sondern die Digitalisierung proaktiv mitzugestalten. Dafür bedarf es neuer Ansätze in Führung und Kommunikation im Kontext des demografischen Wandels.

Jungmann und Kollegen (2015) listen einige Merkmale alternsgerechter Führung auf: Erkennen, dass Altersunterschiede eine Ressource sind, die Wertschätzung der besonderen Fähigkeiten Älterer und das Wissen über ihre möglichen Beeinträchtigungen, eine altersgerechte Gestaltung und Organisation von Arbeit, die Einbindung auch der älteren Mitarbeiter*innen in Weiterbildung, Gesundheits- und Kompetenzförderung v.a. für ältere Beschäftigte und die Organisation der Weitergabe von Wissen.

Junge Führungskräfte bevorzugen die Zusammenarbeit mit Gleichaltrigen. Weitverbreitete Befürchtungen hinsichtlich älterer Arbeitnehmer*innen sind z.B. ,die haben keine Lust mehr auf Veränderung', ,die sind unflexibel' oder ,die nehmen mich nicht ernst' (Matyssek 2011). Eine alternsgerechte Führung sollte dafür sorgen, dass diese Altersstereotype mit ihren negativen Wirkungen auf Leistung und Gesundheit unbedingt vermieden werden. Betriebliche Strategien gegen Altersstereotype, die Führungskräfte initiieren sollten, sind: aktive Reflexion der vorherrschenden Altersbilder im Betrieb, unternehmensinterne Kampagnen zu „Altersbildern“, die aktive Auseinandersetzung mit dem persönlichen Altersbild. Bei Stellenbesetzungen sollten Führungskräfte sich ausschließlich an den individuellen Fähigkeiten statt an vermuteten altersspezifischen Fähigkeiten orientieren. Mitarbeiter*innen jeden Alters sollten also niemals aufgrund ihres Alters, sondern nur aufgrund ihrer Leistung und ihres Engagements beurteilt werden (Initiative Neue Qualität der Arbeit 2017).

Ein Problem in vielen Betrieben ist, dass Ältere kaum noch Feedback von ihren Vorgesetzten bekommen, da diese oft der Meinung sind, der erfahrene ältere Beschäftigte komme allein zurecht oder wolle kein Feedback. Führungskräfte sollten jedoch namentlich den älteren Arbeitnehmer*innen häufiges Feedback geben. Feedback, auch kritisches, ist Wertschätzung. Ältere profitieren stärker als Jüngere von der Unterstützung durch Führungskräfte, weil sie - vor allem unter negativem Altersklima oder durch negative Altersstereotype - an ihren Fähigkeiten zweifeln. Zugleich empfinden sie Unterstützung als besondere Anerkennung und Respekt, deren Bedeutung mit dem Alter zunimmt. Feedback von Kolleg*innen und Vorgesetzten fördert die Selbstwirksamkeit und Innovationskompetenz älterer Beschäftigter (Noefer et al. 2008).

Für den nachhaltigen Erfolg sollte das Gestalten und Führen altersgemischter Teams bestimmte Regeln befolgen. Solche Regeln und Kriterien lassen sich durch Führungskräftetrainings vermitteln (Wegge et al. 2015), 
so dass sich die Wissens- und Erfahrungspools aller Altersgruppen ideal ergänzen.

Eine alternsgerechte Führung bewirkt deutliche Verbesserungen von Wohlbefinden, Gesundheit und Arbeitsfähigkeit älterer Beschäftigter. In einer finnischen Studie zeigte sich, dass die Arbeitsfähigkeit älterer Beschäftigter sich über einen Zeitraum von elf Jahren (51 bis 62 Jahre) um das 3,6-fache verbesserte, wenn die Mitarbeiter"*innen mit dem Verhalten des Vorgesetzten zufrieden waren, im Vergleich zu denen, die unzufrieden waren (Tuomi et al. 1997). Eine Mitarbeiterbefragung von über 14.000 städtischen Angestellten in Deutschland ergab, dass gute Führung die Arbeitsfreude und dadurch die Arbeitsfähigkeit verbesserte; dieser Einfluss war bei den älteren Beschäftigten stärker als bei den jüngeren Erwerbstätigen (Weigl \& Löffler 2014). Für jüngere Menschen sind Aufstiegschancen, Prämien und Events eine Belohnung, für Ältere ist es insbesondere die Würdigung ihrer Erwerbsbiographie (Matyssek 2011). Eine Studie der Bertelsmann-Stiftung (2010) zeigte, dass eine hohe Anerkennung der persönlichen Arbeitsleistung durch Vorgesetzte eine Grundvoraussetzung für die Beschäftigung bis ins Rentenalter darstellt. Aber wie kann dies als „digital Leader" umgesetzt werden?

Führung und Zusammenarbeit in virtuellen Welten prägen inzwischen die Arbeitsrealität. Dabei geht es um die Zusammenarbeit über räumliche, zeitliche und organisationale Grenzen hinweg, bei der Verbindungsnetze mithilfe von Kommunikationstechnologien verwendet werden (Lipnack \& Stamps 1998). Die entfernte Führung findet somit weniger als ,face-toface-Interaktion statt, sondern erfolgt mediengestützt und ist zudem durch kulturelle Unterschiede der Interaktionspartner*innen geprägt. Rund $75 \%$ der virtuellen Teams scheitern aufgrund der hohen Anforderungen an ihre Teammitglieder und vor allem an den Führungskräften (Mummert 2013). Hierbei gilt die De-Lokalisierung mit dem Vertrauensdilemma „Trust needs touch“ als zentrale Herausforderung. Empirische Studien belegen einen Zusammenhang von Vertrauen und Motivation und letztlich auch mit der Leistungsfähigkeit virtueller Teams (Boos et al. 2017). Vier Faktoren (Valenz, Instrumentalität, Vertrauen und Selbstwirksamkeit) spielen nach dem VIST-Modell von Hertel (2002) eine entscheidende Rolle zur Erhöhung der Arbeitsmotivation in virtuellen Teams. Werden diese Komponenten von den einzelnen Teammitgliedern als stark ausgeprägt erlebt, so sagt das Modell eine hohe Motivation zur Arbeit im Team voraus. Ist eine Komponente oder sind mehrere Komponenten jedoch niedrig ausgeprägt, so kann die Arbeitsmotivation darunter leiden. Führungskräfte sollten alle Faktoren berücksichtigen, Transparenz schaffen und regelmäßige Einzel- und Teamfeedbacks geben, um die Motivati- 
on zu fördern. Sie müssen regelmäßige gemeinsame Treffen - computergestützt via Video- oder Webkonferenz - schaffen und sich dafür die nötige Medienkompetenz aneignen. Für den Vertrauensaufbau ist eine gemeinsame Veranstaltung zum Projekt- oder Teamstart sinnvoll, da ein solcher „Kick-off“ auch informellen Austausch und den Aufbau persönlicher Beziehungen ermöglicht. Alternsgerechte Führung - auch über die Distanz muss und kann man lernen. Betriebliche Maßnahmen sind hier das Erlernen von Wissen über Altersveränderungen sowie eines sensiblen und rücksichtsvollen Umgangs mit älteren Beschäftigten oder altersgemischten Gruppen, welcher in Weiterbildungskursen für Führungskräfte erlernbar ist (Ries et al. 2013; Wegge et al. 2015). An solchen Schulungen sollten auch Führungskräfte der mittleren und unteren Ebene teilnehmen. Zudem ist eine obligatorische Teilnahme anzuraten, da sonst die Gefahr besteht, dass an solchen Weiterbildungen hauptsächlich bereits sensibilisierte und engagierte Führungskräfte teilnehmen. Hinsichtlich der Führung auf Distanz sind zunehmend weitere Kompetenzbereiche erforderlich, u.a. Kommunikationsfähigkeit in virtuellen Welten, IT-Kompetenzen, digitale Kompetenzen, Motivationsfähigkeit oder kulturkongruente Führungsstile. Bei der Entwicklung von neuen Führungskräfteschulungen sollten Betriebe im Dialog mit der Managementebene gemeinsam Lösungsversuche wagen, um eine gemeinsame Führungskultur im digitalen Zeitalter entstehen zu lassen.

\section{Fazit und Ausblick}

Moderne digitalisierte Arbeit fordert den Beschäftigten ein hohes Maß an Motivation, Lernfähigkeit, und kognitiven Fähigkeiten ab. Ältere können neben hohen Kompetenzen auch Einschränkungen in verschiedenen sensorischen, psychomotorischen und v.a. in manchen kognitiven Funktionen zeigen. Insbesondere kognitive Veränderungen hängen von externen Einflussfaktoren ab, wie v.a. der Art der Arbeit. Daher sollten neben verhaltensorientierten (Trainings-) Maßnahmen gestalterische und organisatorische Maßnahmen getroffen werden, die an diesen Faktoren ansetzen. Im Bereich der Verhältnisprävention sind dies eine alter(n)sfreundliche Gestaltung des Arbeitsplatzes und der Arbeit selbst, und insbesondere eine altersgerechte Führung. Hierzu bedarf es einer intensiven Schulung von Führungskräften. In Kombination mit verhaltenspräventiven Maßnahmen lässt es sich so erreichen, dass ältere Beschäftigte auch in der digitalisierten Arbeitswelt bis zum Rentenalter gesund und leistungsfähig bleiben. 
Der Einfluss der Arbeit auf die altersbegleitende Abnahme kognitiver Funktionen wurde bereits von Andreas Kruse beim Kongress Altern und Arbeit im Jahr 2000 (von Rothkirch 2000) hervorgehoben. Die disziplinübergreifende Forschung der vergangenen 20 Jahre zeigt, dass Beschäftigte durch zielgruppenspezifische und bedarfsgerechte Maßnahmen im betrieblichen Setting physisch und psychisch gesund altern können. Insbesondere konnte durch neurowissenschaftliche Forschungen gezeigt werden, dass günstige Arbeitsbedingungen wie kurz- und längerfristige Arbeitswechsel die mentale Fitness älterer Beschäftigter deutlich verbessern, was sich nicht nur in Testleistungen, sondern in neuronalen Strukturen und Funktionen zeigt. Auf der gleichen Tagung bestätigte Ilmarinen, dass eine Kombination der wichtigsten betrieblichen Maßnahmen die Arbeitsfähigkeit älterer Mitarbeiter*innen konstant hält oder gar steigert. In den letzten 20 Jahren konnte durch moderne Methoden gezeigt werden, dass Interventionen bei älteren Beschäftigten auch neuronale Veränderungen und dadurch Leistungsverbesserungen bewirken. Vor allem sollte die wachsende Gruppe der über 60-Jährigen auch gegen Ende der Berufslaufbahn motiviert werden, was durch das Zusammenspiel von altersgerechter Arbeitsgestaltung, Weiterbildung und Führung erreicht werden kann. Daneben müssen gerade die über 60-Jährigen weit stärker als bisher angeregt werden, günstige Lebensstilmaßnahmen wie tägliche Bewegung zu ergreifen. Trotz einschlägiger wissenschaftlicher Erkenntnisse durch zahlreiche Studien und Publikationen gelingt die Transferleistung in die Praxis nur unzureichend. Insbesondere aufgrund der stetigen Veränderungen der Arbeit muss dem Themenbereich alters- und alternsgerechtes Arbeiten ein höherer Stellenwert in Gesellschaft, Politik und Wirtschaft zugewiesen werden. Dieses Ziel kann durch beharrliche Wissensvermittlung zum Thema Alter und Arbeit in den Betrieben und vor politischen Gremien erreicht werden. Nur so gelingt langfristig eine proaktive Bewältigung der heutigen und zukünftigen Herausforderungen. 


\section{Literatur}

Bertelsmann-Stiftung (2010). Vorgesetzte können Burnout am Arbeitsplatz reduzieren. Sozial unterstützendes Verhalten gehört zu moderner Personalpolitik. Pressemitteilung. Berlin/Gütersloh, 18.05.2010.

Boos, M., Hardwig, T., Rietmüller, M. (2017). Führung und Zusammenarbeit in verteilten Teams. Praxis der Personalpsychologie. Human Resource Management kompakt. Band 35. Göttingen: Hogrefe.

Bundesanstalt für Arbeitsschutz und Arbeitsmedizin, BAuA (Hrsg.) (2012). Länger geistig fit im Beruf! Abschlussbericht PFIFF 2: Umsetzung des Programms PFIFF - Training zur Förderung von Gesundheit und geistiger Leistungsfähigkeit bei älteren Arbeitnehmern. Dortmund: BAuA (Initiative Neue Qualität der Arbeit, INQA-Bericht, Nr. 43).

Falkenstein, M, Gajewski, P., Stahn, C., Zülch, J. (2020). Stressmanagement und kognitives Training zur Förderung kognitiver und emotionaler Fähigkeiten älterer Beschäftigter in der Automobilfertigung. In: M. Falkenstein, C. Kardys (Hrsg.), Arbeit, Kognition und Alter. Stuttgart: Kohlhammer.

Falkenstein, M. (2013). Menschengerechtes Arbeiten für ältere Beschäftigte. Zeitschrift für betriebliche Prävention und Unfallversicherung (BPUVZ), April 2013, 210-215.

Falkenstein, M., Kardys, C. (Hrsg.) (2020). Arbeit, Kognition und Alter. Stuttgart: Kohlhammer.

Falkenstein, M., Kardys, C. (2018). Auswirkungen von Arbeitsstress auf eine alternde Belegschaft. Arbeitsmedizin Sozialmedizin Umweltmedizin 53, 592-595.

Falkenstein, M., Gajewski, P., Kardys, C. (2017). Wie lassen sich Beschäftigte in (geistiger) Bewegung halten? In: G. Richter, C. Hecker, A. Hinz (Hrsg.), Produktionsarbeit in Deutschland - mit alternden Belegschaften. Berlin: Erich Schmidt Verlag.

Falkenstein, M. (2017). Die Förderung der fluiden Intelligenz bei Beschäftigten als Voraussetzung für Gesundheit und Beschäftigungsfähigkeit. In: W. Jochmann, I. Böckenholt, S. Diestel (Hrsg.), HR-Exzellenz. Innovative Ansätze in Leadership und Transformation. Wiesbaden: Springer Gabler.

Falkenstein, M. (2012). Lernen im Alter. In: C. Kreklau, J. Siegers (Hrsg.), Handbuch der Aus- und Weiterbildung. Köln: Wolters Kluwer.

Falkenstein, M., Hoormann, J., Hohnsbein, J. (2001). Changes of error-related ERPs with age. Exp. Brain Res. 138, 258-262.

Freude, G., Falkenstein, M., Zülch, J. (2010). Förderung und Erhalt intellektueller Fähigkeiten für ältere Arbeitnehmer. Abschlussbericht des Projekts "Pfiff". Dortmund: Initiative Neue Qualität der Arbeit (INQA-Bericht, Nr. 39), http://w ww.inqa.de/Inqa/Navigation/publikationen,did=255306.html.

Getzmann, S., Gajewski, P.D., Falkenstein, M. (2013). Does age increase auditory distraction? Electrophysiological correlates of high and low performance in seniors. Neurobiology of Aging 34(8), 1952-62. 
Godde, B., Oltmanns, J., Richter, G., Voelcker-Rehage, C., Staudinger, U. M. (2020). Arbeits- und Karrieregestaltung zur Förderung der kognitiven Entwicklung im Erwachsenen- und höheren Alter. In: M. Falkenstein, C. Kardys (Hrsg.), Arbeit, Kognition und Alter. Stuttgart: Kohlhammer.

Hacker, W. (2004). Leistungs- und Lernfähigkeiten älterer Menschen. In: M. v. Cranach, H.-D. Schneider, R. Winkler \& E. Ulich (Hrsg.), Ältere Menschen im Unternehmen. Chancen, Risiken, Modelle. Bern: Haupt, 163-172.

Hertel, G. (2002). Management virtueller Teams auf der Basis sozialpsychologischer Theorien: Das VIST Modell. In: E. H. Witte (Hrsg.), Sozialpsychologie wirtschaftlicher Prozesse. Lengerich: Pabst, 172-202.

Ilmarinen, J., Tempel, J. (2013). Arbeitsleben 2025. Hamburg: VSA-Verlag.

Initiative Neue Qualität der Arbeit (INQA) (2017): Alle in eine Schublade? Altersstereotype erkennen und überwinden. Dortmund, https:/www.inqa.de/Shared Docs/PDFs/DE/Publikationen/altersstereotype-erkennen.pdf?_blob=publicatio $n F i l e \& v=4$.

Jungmann, F., Bilinska, P., \& Wegge, J. (2015). Alter(n)sgerechte Führung. In: J. Felfe (Hrsg.), Trends der psychologischen Führungsforschung: Neue Konzepte, Methoden und Erkenntnisse (Psychologie für das Personalmanagement). Göttingen: Hogrefe.

Kardys, C., Getzmann, S., Falkenstein, M. (2020). Körperliches und kognitives Training zur Förderung kognitiver Fähigkeiten bei Beschäftigten in der Automobilfertigung. In: M. Falkenstein, C. Kardys (Hrsg.), Arbeit, Kognition und Alter. Stuttgart: Kohlhammer.

Lipnack, J., Stamps, J. (1998). Virtuelle Teams: Projekte ohne Grenzen; Teambildung, virtuelle Orte, intelligentes Arbeiten, Vertrauen in Teams. Redline Wirtschaftsverlag.

Lohmann-Haislah, A. (2013). Stressreport Deutschland 2012. Psychische Anforderungen, Ressourcen und Befinden. Berlin: Bundesanstalt für Arbeitsschutz und Arbeitsmedizin.

Marquié, J. C., Duarte, L. R., Bessières, P., Dalm, C., Gentil, C., Ruidavets, J. B. (2010). Higher mental stimulation at work is associated with improved cognitive functioning in both young and older workers. Ergonomics 53(11), 1287-301.

Matyssek, A. K. (2011). Gesund führen - sich und andere! Trainingsmanual zur psychosozialen Gesundheitsförderung im Betrieb. 1. Aufl. Norderstedt: bod.

Mummert, R. (2013). Management 2.0: Drei von vier virtuellen Teams scheitern, https://www.rochusmummert.com/downloads/news/67_130416_rochus_mum mert_virtuelle_teams.pdf (Zugriff am 14.06.2020).

Mühlenbrock, I. (2016). Alterns- und altersgerechte Arbeitsgestaltung. Grundlagen und Handlungsfelder für die Praxis. Herausgeber: Bundesanstalt für Arbeitsschutz und Arbeitsmedizin (BAuA). 
Noefer, K., Stegmaier, R., Molter, B., Sonntag, K. (2008). Entwicklungsbezogene Selbstwirksamkeit als Mediator für den Zusammenhang von Feedback und Ideenimplementierung. Zeitschrift für Arbeits- und Organisationspsychologie 52(2), N.F. 26, 61-69.

Otte, H. R., Seifritz, E. (2014). Bis er uns umbringt? Wie Stress die Gesundheit attackiert - und wie wir uns schützen können. Bern: Huber.

Ries, B. C., Diestel, S., Shemla, M., Liebermann, S. C., Jungmann F., Wegge, J., Schmidt, K. H. (2013). Age Diversity and Team Effectiveness. In: C. M. Schlick, E. Frieling, J. Wegge (Hrsg), Age-Differentiated Work Systems. Berlin: Springer, 89-118.

Rodriguez, F., Spilski, J. (2020). Mentale Anforderungen am Arbeitsplatz als Schutz gegen Demenz? In: M. Falkenstein \& C. Kardys (Hrsg.), Arbeit, Kognition und Alter. Stuttgart: Kohlhammer.

Stamov Roßnagel, C. (2008). Mythos „alter“ Mitarbeiter. Lernkompetenz jenseits der 40?! Weinheim: Beltz PVU.

Tuomi, K., Ilmarinen, J., Seitsamo, J., Huuhtanen, P., Martikainen, R., Nygård, C. H., Klockars, M. (1997). Summary of the Finnish research project (1981-1992) to promote the health and work ability of aging workers. Scandinavian Journal of Work and Environmental Health, Suppl 23(1), 66-71.

Vieluf, S., Voelcker-Rehage, C., Godde, B. (2020). Altersbedingte Veränderungen sensomotorischer Koordination und Handgeschicklichkeit im Arbeitskontext. In: M. Falkenstein, C. Kardys (Hrsg.), Arbeit, Kognition und Alter. Stuttgart: Kohlhammer.

von Rothkirch, C. (Hrsg.) (2000). Altern und Arbeit: Herausforderung für Wirtschaft und Gesellschaft. Beitrage, Diskussionen und Ergebnisse eines Kongresses mit internationaler Beteiligung. Berlin: Ed. Sigma.

Wegge, J., Jungmann, F. (2015). Altersgemischte Teamarbeit. Ein Training für die bessere Zusammenarbeit von Jung und Alt in Arbeitsgruppen. BPUVZ 127, 116-120.

Weigl, M., Löffler, M. (2014). Erhalt der Arbeitsfähigkeit älterer Beschäftigter: Ergebnisse und Zusammenhänge zu Arbeitsfähigkeit und psychosozialen Merkmalen der Arbeit bei städtischen Angestellten. Berlin, 26.11.14, DGPPN Kongress 2014.

Weißbecker-Klaus, X. (2014). Multitasking und Auswirkungen auf die Fehlerverarbeitung. Forschung Projekt F 2247. Berlin: Bundesanstalt für Arbeitsschutz und Arbeitsmedizin (BAuA).

Wild-Wall, N., Gajewski, P. D., Falkenstein, M. (2009). Kognitive Leistungsfähigkeit älterer Arbeitnehmer. Zeitschrift für Gerontologie und Geriatrie 42, 299-304. 
